\title{
30th AND 31st ANNUAL SASKATCHEWAN CHRISTMAS MAMMAL COUNTS - 2002 AND 2003
}

ALAN R. SMITH, 115 Perimeter Road, Saskatoon SK S7N 0X4.

\section{Introduction}

I would like to apologize to readers for the delay in presenting the results of the 30th and 31st Saskatchewan Christmas Mammal Counts (CMCs) for 2002 and 2003. It is a testament to the hard work of the previous compiler, the late Wayne Harris, that for 13 years he was able to compile the results of both the Christmas Bird and Mammal Counts and present them in timely fashion for publication in Blue Jay.

In order to partially redress this delay in what has become, in its own right, an important activity of members of Nature Saskatchewan, I have not only compiled the results of these last two counts, but I have summarized the results of the last 31 years of mammal counts. Readers should note that some changes have been made in the presentation of the mammal counts from previous years. This was done to harmonize them with the bird counts, and to simplify the task of tabulating an increasingly large number of counts. The number of mammals actually seen (or, more rarely, heard) on count day is recorded separately from those detected by other means or recorded during the count period but not on count day. Thus mammals detected on count day on the basis of tracks are indicated with the letter " $t$ ", those by other means (dead animals or clearly identifiable chewing, digging, dens or lodges) by the letter "o", those recorded during the count period but not on count day by the letter "c".

For weather, coverage and participants for the 2002 CMC, I refer readers to the March
2003 issue of Blue Jay, (Vol. 61, No. 1) and for the 2003 count to the March 2004 issue (Vol. 62, No. 1).

The 30th Christmas Mammal Count-2002

Counts (Table 1) were received from 89 localities, which is down from the record high of 102 set in 2001 . The 33 species seen on count day tied the 1991 record. Indian Head had the most species seen or heard on count day (13), while honours for the most species present over the entire count period (15) were shared between Candle Lake and Prince Albert National Park.

The most abundant mammal was the White-tailed Deer, closely followed by its cousin the Mule Deer. The American Red Squirrel and Pronghorn placed third and fourth. The most frequently seen mammals were the White-tailed Deer seen on $60 \%$ of all counts, Coyote on 56\%, American Red Squirrel on $55 \%$, and Mule Deer on $30 \%$. While Mule Deer were much less often seen than were white-tails, they occurred in larger numbers when they were seen.

The only new species in 2002, and the first since 1996, was a jumping mouse, either a Meadow Jumping Mouse or Western Jumping Mouse. The species was noted on the basis of its distinctive tracks on the Clark's Crossing count. Also noteworthy was the Richardson's Ground Squirrel seen on the Saskatoon count. The eight Mule Deer seen on the Porcupine Plain count were at the northeastern limit of their range. The 73 American Red Squirrels tallied on the Fort 
Walsh count may have been a record high count for the species on a CMC.

\section{The 31st Christmas Mammal Count-2003}

Ninety-one counts were completed in 2003 (Table 2.), two more than in 2002. The 35 species seen on count day set a new record. Indian Head had the most species seen or heard on count day (14), while Regina recorded 16 species over the count period.

The three most abundant species were no surprise: White-tailed Deer followed by Mule Deer and American Red Squirrel. However, Elk, as the fourth most common species, was a surprise. The four species most frequently seen were the same as in 2002. White-tailed Deer were seen on $69 \%$ of all counts, American Red Squirrel on $58 \%$, Coyote on $52 \%$, and Mule Deer on $30 \%$.

Two new species, Thirteen-lined Ground Squirrel and Least Chipmunk, were added on this count. Although Thirteen-lined Ground Squirrels were seen during the count period in 1987 and 1991, the 2003 sighting from Craven was the first sighting on count day. Chipmunks were reported twice on count day. One was seen at Archerwill, and tracks were noted at Saskatoon. Mammal counters from Craven seemed to be going "squirrelly" as they also counted four of each of the tree squirrels, the Grey, Fox and American Red.

\section{Summary of Christmas Mammal Counts 1973-2003}

Wayne Harris began compiling the information on mammals counted incidentally on Christmas Bird Counts (CBCs) beginning in 1973 (Table 3). At that time, eleven mammal species were reported from eight of $46 \mathrm{CBCs}$ published in Blue Jay. ${ }^{1}$ Interest rapidly grew; by the end of the decade, over $70 \%$ of CBCs had a CMC as well. By the mid-1990s, the figure had risen to over $90 \%$. To date, 1,972 mammal counts have been conducted in almost 300 localities.

According to Wapple, 79 species of wild mammals have been recorded in Saskatchewan. ${ }^{2}$ The Christmas Mammal Count has provided evidence for the occurrence of 55 species (feral horses and cats are excluded). Of these, 49 species have actually been seen on count day. One other species, the American Water Shrew, has been seen during the count period, three others have been recorded on the basis of dead animals, and two others have been recorded only on the basis of tracks.

Although the data from the CMCs have not yet been subjected to a statistical analysis, a cursory examination of data from the last 32 years suggests that there have been major changes in the province's mammal population over this period. Among the more obvious and interesting are:

1. The arrival and spread of the Fox Squirrel. 2. Increasing appearance of ground squirrels, Black-tailed Prairie Dogs and Least Chipmunks. These species should be hibernating during the count period. Their appearance on CMCs may be an indication of a warming climate.

3. The increase in the populations of all the ungulates, and the spread of Moose and Elk onto the plains.

Thanks to the foresight of Wayne Harris, we now have information with which to conduct more detailed analysis of the population trends and distributional changes of Saskatchewan mammals.

1. HARRIS, W.C. 1974. Saskatchewan Christmas Mammal Counts, 1973. Blue Jay 32(2):115.

2. WAPPLE, R.D. 1999. Mammals. In: Fung, K. (ed.) Atlas of Saskatchewan. University of Saskatchewan, Saskatoon. Pp 139-142. 
Table 1. $30^{\text {th }}$ Saskatchewan Christmas Mammal Count-2002.

Species

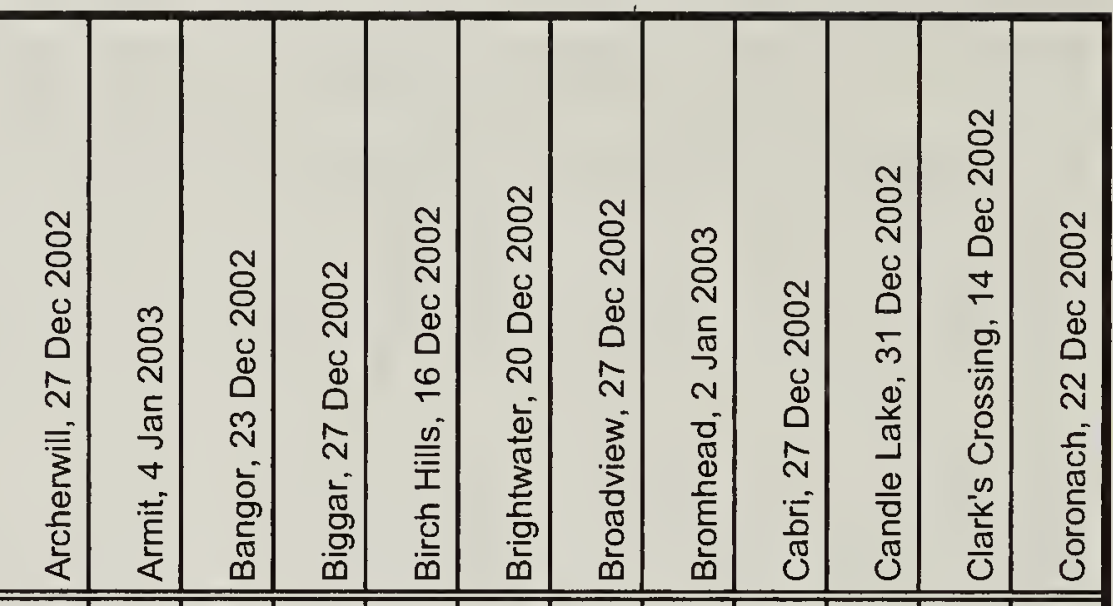

Shrew species

Nuttall's Cottontail

Snowshoe Hare

White-Tailed Jack Rabbit

Richardson's Ground Squirrel

Black-Tailed Prairie Dog

Grey Squirrel

Fox Squirrel

American Red Squirrel

Northern Flying-Squirrel

American Beaver

Deer Mouse

Muskrat

Meadow Vole

Vole species

Jumping Mouse species

Mouse species

Norway Rat

House Mouse

American Porcupine

Coyote

Wolf

Red Fox

Raccoon

American Marten

Fisher

Ermine

Long-tailed Weasel

Least Weasel

Weasel species

American Mink

American Badger

Striped Skunk

River Otter

Lynx

Woodland Caribou

Mule Deer

White-tailed Deer

Deer species

Moose

Elk

Moose/Elk

Pronghorn

Totals seen/heard on count day

Total species seen/heard

Total species recorded by tracks

Total species otherwise recorded

Species recorded count period

Total species count period and day

\begin{tabular}{rr|r|r|r|r|r|r|r|r|r|r|r|} 
& & & & & $\mathrm{t}$ & & & & & & & \\
\hline & & & & & & & & & & & & 0 \\
\hline & & & 3 & & & & & 2 & & & 2 & \\
\hline
\end{tabular}


Table $1.30^{\text {th }}$ Saskatchewan Christmas Mammal Count-2002.

Species

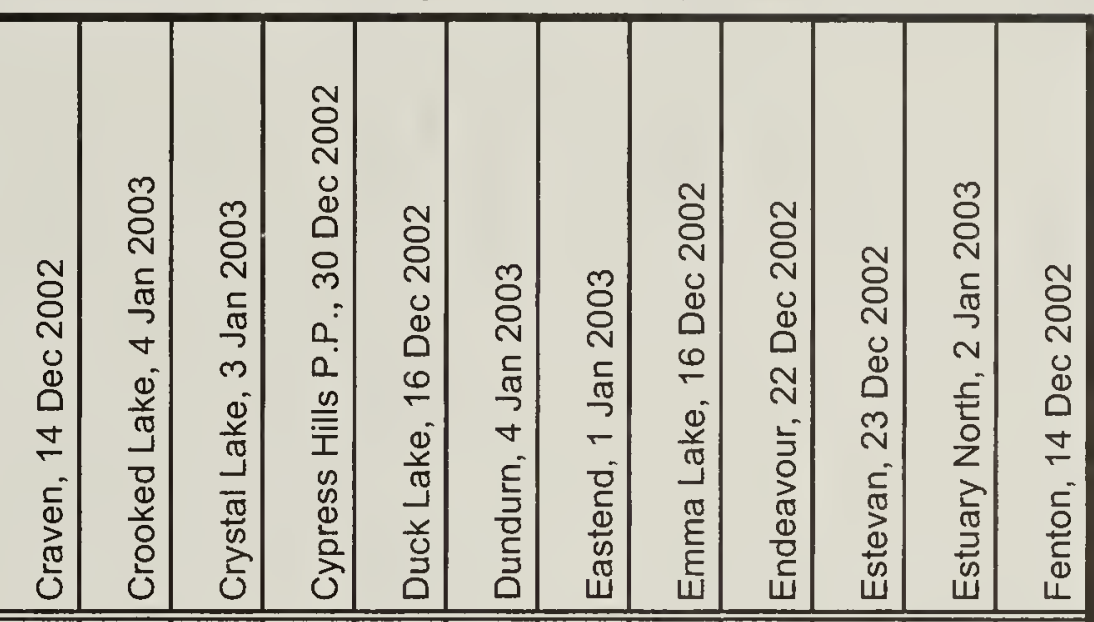

Shrew species

Nuttall's Cottontail

Snowshoe Hare

White-Tailed Jack Rabbit

Richardson's Ground Squirrel

Black-Tailed Prairie Dog

Grey Squirrel

Fox Squirrel

American Red Squirrel

Northern Flying-Squirrel

American Beaver

Deer Mouse

Muskrat

Meadow Vole

Vole species

Jumping Mouse species

Mouse species

Norway Rat

House Mouse

American Porcupine

Coyote

Wolf

Red Fox

Raccoon

American Marten

Fisher

Ermine

Long-tailed Weasel

Least Weasel

Weasel species

American Mink

American Badger

Striped Skunk

River Otter

Lynx

Woodland Caribou

Mule Deer

White-tailed Deer

Deer species

Moose

Elk

Moose/Elk

Pronghorn

Totals seen/heard on count day

Total species seen/heard

Total species recorded by tracks

Total species otherwise recorded

Species recorded count period

Total species count period and day

\begin{tabular}{ll|l|l|} 
& & & \\
\hline & & & \\
& & &
\end{tabular}

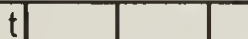

\section{.}


Table 1.30 ${ }^{\text {th }}$ Saskatchewan Christmas Mammal Count-2002.

Species

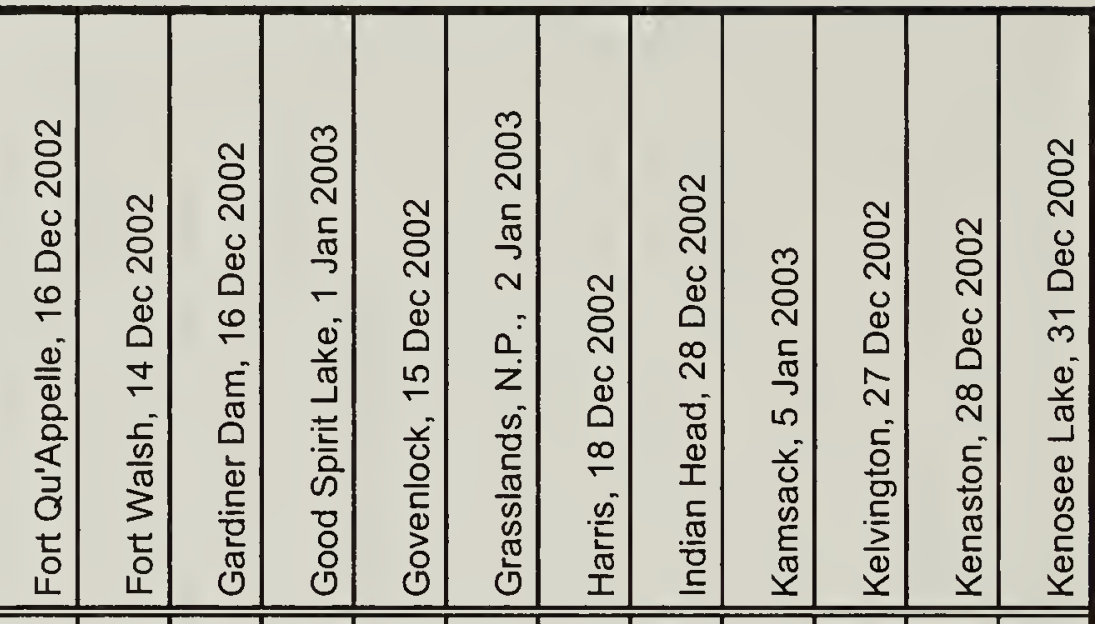

Shrew species

Nuttall's Cottontail

Snowshoe Hare

White-Tailed Jack Rabbit

Richardson's Ground Squirrel

Black-Tailed Prairie Dog

Grey Squirrel

Fox Squirrel

American Red Squirrel

Northern Flying-Squirrel

American Beaver

Deer Mouse

Muskrat

Meadow Vole

Vole species

Jumping Mouse species

Mouse species

Norway Rat

House Mouse

American Porcupine

Coyote

Wolf

Red Fox

Raccoon

American Marten

Fisher

Ermine

Long-tailed Weasel

Least Weasel

Weasel species

American Mink

American Badger

Striped Skunk

River Otter

Lynx

Woodland Caribou

Mule Deer

White-tailed Deer

Deer species

Moose

Elk

Moose/Elk

Pronghorn

\begin{tabular}{l|r|r|r|r|r|r|}
\hline & 1 & & & & & \\
\hline & 3 & 1 & & 1 & 3 &
\end{tabular}

1

. 
Table 1. $30^{\text {th }}$ Saskatchewan Christmas Mammal Count-2002.

Species

\begin{tabular}{|c|c|c|c|c|c|c|c|c|c|c|c|}
\hline 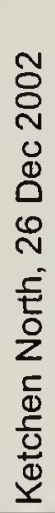 & 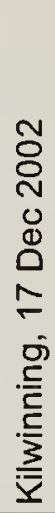 & 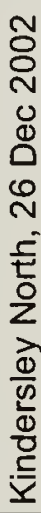 & 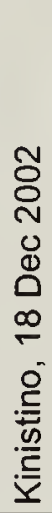 & 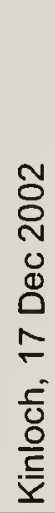 & 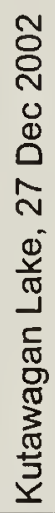 & 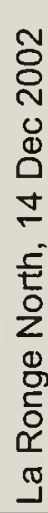 & 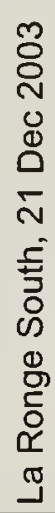 & 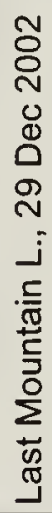 & 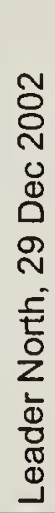 & 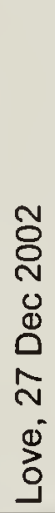 & $\begin{array}{l} \\
0 \\
0 \\
0 \\
0 \\
0 \\
0 \\
0\end{array}$ \\
\hline
\end{tabular}

Shrew species

Nuttall's Cottontail

Snowshoe Hare

White-Tailed Jack Rabbit

Richardson's Ground Squirrel

Black-Tailed Prairie Dog

Grey Squirrel

Fox Squirrel

American Red Squirrel

Northern Flying-Squirrel

American Beaver

Deer Mouse

Muskrat

Meadow Vole

Vole species

Jumping Mouse species

Mouse species

Norway Rat

House Mouse

American Porcupine

Coyot

Wolf

Red Fox

Raccoon

American Marten

Fisher

Ermine

Long-tailed Weasel

Least Weasel

Weasel species

American Mink

American Badger

Striped Skunk

River Otter

Lynx

Woodland Caribou

Mule Deer

\begin{tabular}{l|l|l|l}
4 & $\mathrm{t}$ & 9
\end{tabular}

White-tailed Deer

Deer species

Moose

Elk

Moose/Elk

Pronghorn

Totals seen/heard on count day

Total species seen/heard

17

\begin{tabular}{r|r|r|r}
\hline$t$ & & & \\
\hline & & $t$
\end{tabular}

\begin{tabular}{r|r|r|r|r} 
& & & 6 & \\
$\mathrm{t}$ & $\mathrm{t}$ & & &
\end{tabular}

t

Total species recorded by tracks

Total species otherwise recorded

Species recorded count period

Total species count period and day 
Table 1. 30 ${ }^{\text {th }}$ Saskatchewan Christmas Mammal Count-2002.

Species

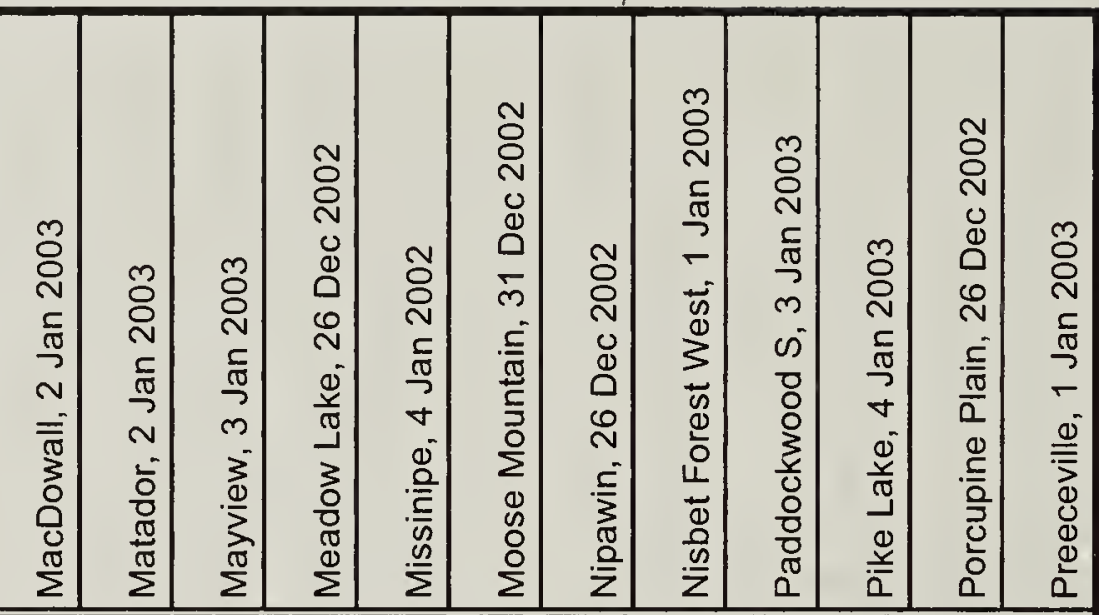

Shrew species

Nuttall's Cottontail

Snowshoe Hare

White-Tailed Jack Rabbit

Richardson's Ground Squirrel

Black-Tailed Prairie Dog

Grey Squirrel

Fox Squirrel

American Red Squirrel

Northern Flying-Squirrel

American Beaver

Deer Mouse

Muskrat

Meadow Vole

Vole species

Jumping Mouse species

Mouse species

Norway Rat

House Mouse

American Porcupine

Coyote

Wolf

Red Fox

Raccoon

American Marten

Fisher

Ermine

Long-tailed Weasel

Least Weasel

Weasel species

American Mink

American Badger

Striped Skunk

River Otter

Lynx

Woodland Caribou

Mule Deer

White-tailed Deer

Deer species

Moose

Elk

Moose/Elk

Pronghorn

Totals seen/heard on count day

Total species seen/heard

Total species recorded by tracks

\begin{tabular}{l|l|} 
& \\
$\mathrm{t}$ &
\end{tabular}

t

\section{$+2$}

(1)

\section{(2)}

\begin{tabular}{|r|r|r|r|r|r|r|r|r|r|r|r|}
\hline $\mathrm{t}$ & & & $\mathrm{t}$ & $\mathrm{t}$ & & $\mathrm{t}$ & & 1 & & $\mathrm{t}$ & \\
\hline $\mathrm{t}$ & $\mathrm{t}$ & & & & & & & $\mathrm{t}$ & & & $\mathrm{t}$ \\
\hline & & & & & & & & & & & \\
\hline & & & & & & & & & & & \\
\hline
\end{tabular}

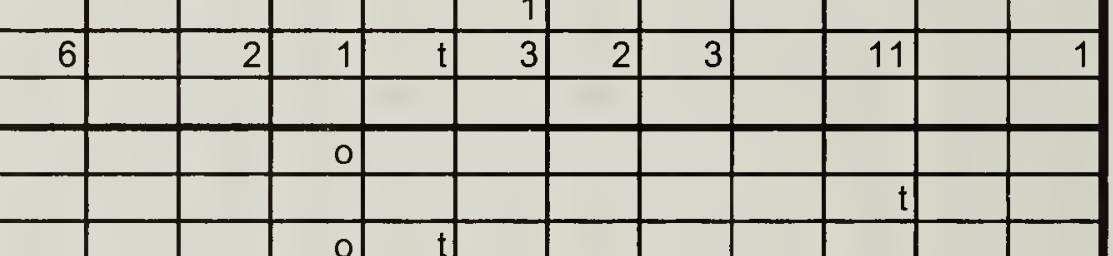

Total species otherwise recorded

Species recorded count period

Total species count period and day

\begin{tabular}{rr|r|r|r|l}
\hline & & & 0 & $\mathrm{t}$ & \\
\hline $\mathrm{t}$ & & & & & \\
& & & & & \\
\hline & & & $\mathrm{t}$ & & \\
\hline $\mathrm{t}$ & & & & & \\
$\mathrm{t}$ & 10 & & & & \\
\hline
\end{tabular}

3

\begin{tabular}{rl|r|r|r|r|l|l|r|r|r|r|r|}
\hline & & & $\mathrm{t}$ & $\mathrm{t}$ & & & 1 & 5 & 15 & $\mathrm{t}$ & 20 \\
\hline $\mathrm{t}$ & & & $\mathrm{t}$ & & & & 1 & & $\mathrm{t}$ & & 1 \\
\hline & & & & & & & & & & & \\
\hline
\end{tabular}


Table 1. $30^{\text {th }}$ Saskatchewan Christmas Mammal Count-2002.

Species

\begin{tabular}{|c|c|c|c|c|c|c|c|c|c|c|c|}
\hline 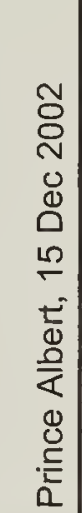 & 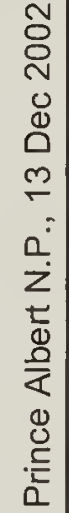 & 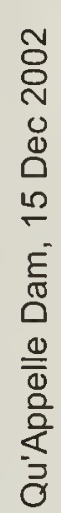 & 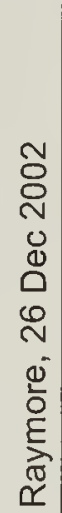 & 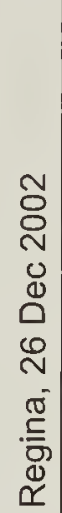 & 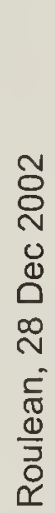 & 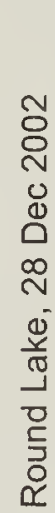 & 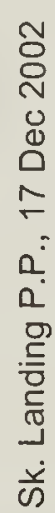 & 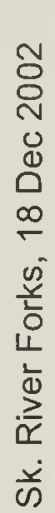 & 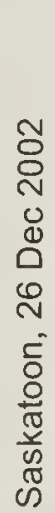 & 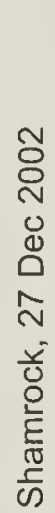 & 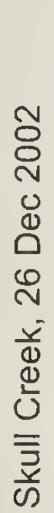 \\
\hline
\end{tabular}

Shrew species

Nuttall's Cottontail

Snowshoe Hare

White-Tailed Jack Rabbit

Richardson's Ground Squirrel

Black-Tailed Prairie Dog

Grey Squirrel

Fox Squirrel

American Red Squirrel

Northern Flying-Squirrel

American Beaver

Deer Mouse

Muskrat

Meadow Vole

Vole species

Jumping Mouse species

Mouse species

Norway Rat

House Mouse

American Porcupine

Coyote

Wolf

Red Fox

Raccoon

American Marten

Fisher

Ermine

Long-tailed Weasel

Least Weasel

Weasel species

American Mink

American Badger

Striped Skunk

River Otter

Lynx

Woodland Caribou

Mule Deer

White-tailed Deer

Deer species

Moose

Elk

\begin{tabular}{lr|r|r|r|r|r|r|r|r|r|r|} 
& & & & & & & & & & & \\
$\mathrm{t}$ & $\mathrm{t}$ & 3 & $\mathrm{t}$ & $\mathrm{t}$ & & $\mathrm{t}$ & & $\mathrm{t}$ & 2 & & \\
\hline
\end{tabular}

Moose/Elk

Pronghorn

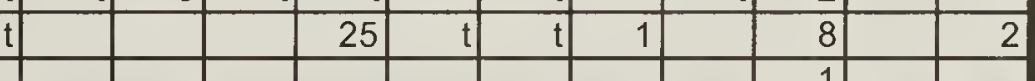

Totals seen/heard on count day

\begin{tabular}{|l|l|l|l|}
\hline & & & \\
\hline & & & 2 \\
\hline
\end{tabular}

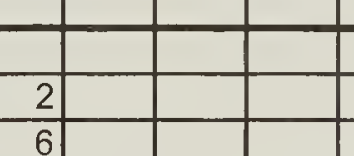

\begin{tabular}{r|r|r|r|}
\hline & 1 & \\
\hline & & \\
\hline
\end{tabular}

\begin{tabular}{|l|l|l|l|r|r|r|r|r|r|}
\hline 35 & & & & 4 & & 12 & 3 & & 2 \\
\hline
\end{tabular}

Total species seen/heard

Total species recorded by tracks

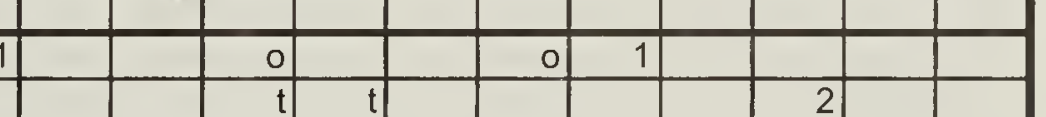

Total species otherwise recorded

Species recorded count period

Total species count period and day

\begin{tabular}{r|r|r|r|r|r}
\hline 1 & & & 0 & \\
\hline & & & & $\mathrm{t}$ & \\
\hline & & & 1 & & \\
\hline
\end{tabular}

t

\begin{tabular}{|r|r|r|r|r|r}
\hline & 1 & & & 2 \\
\hline & & & & \\
\hline
\end{tabular}

\begin{tabular}{l|r|r|}
\hline & & \\
\hline & 3 &
\end{tabular}

$+$

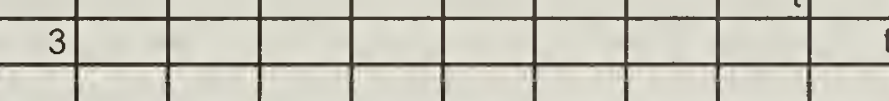

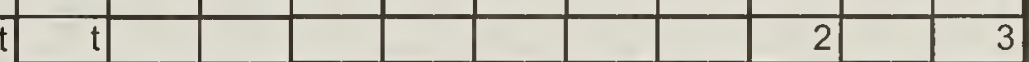

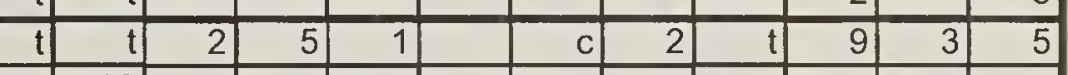

\begin{tabular}{|r|r|r|r|r|r|r}
\hline 2 & & & & & \\
\hline
\end{tabular}

\begin{tabular}{|l|l|l|l|l|}
\hline & & $\mathrm{t}$ & 1 & 1 \\
\hline & & & & $\mathrm{t}$ \\
\hline
\end{tabular}

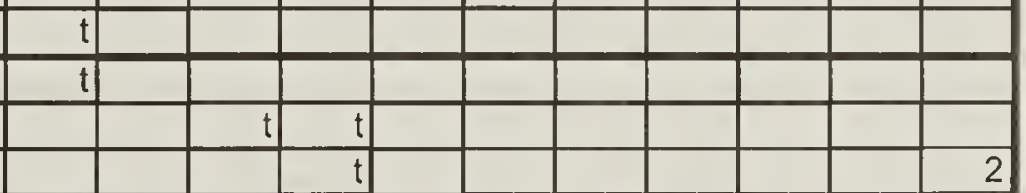


Table 1.30 ${ }^{\text {th }}$ Saskatchewan Christmas Mammal Count-2002.

Species

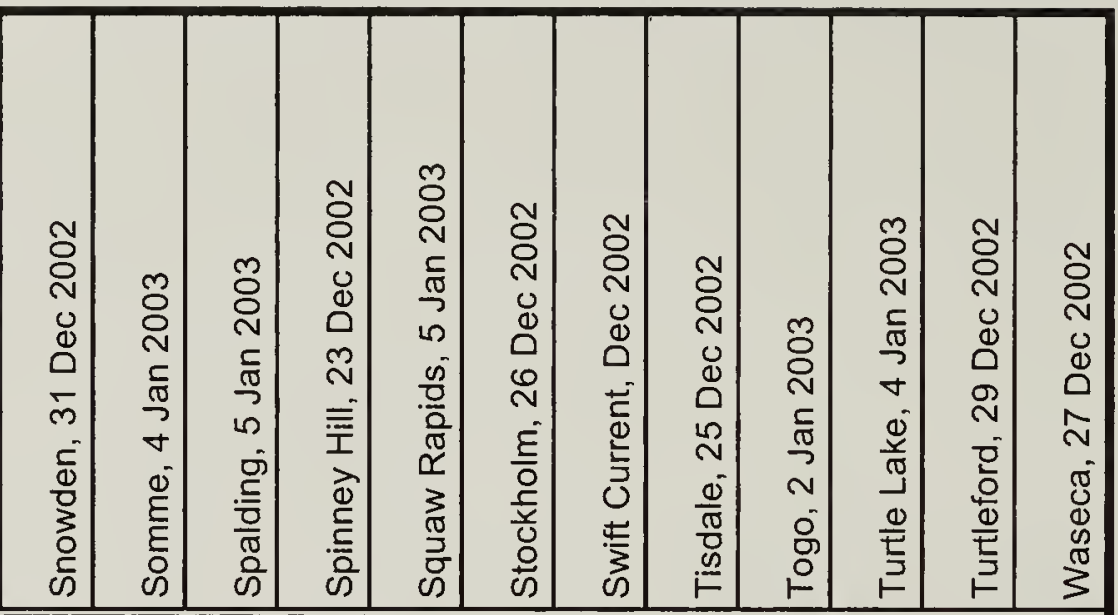

Shrew species

Nuttall's Cottontail

Snowshoe Hare

White-Tailed Jack Rabbit

Richardson's Ground Squirrel

Black-Tailed Prairie Dog

Grey Squirrel

Fox Squirrel

American Red Squirrel

Northern Flying-Squirrel

American Beaver

Deer Mouse

Muskrat

Meadow Vole

Vole species

Jumping Mouse species

Mouse species

Norway Rat

House Mouse

American Porcupine

Coyote

Wolf

Red Fox

Raccoon

American Marten

Fisher

Ermine

Long-tailed Weasel

Least Weasel

Weasel species

American Mink

American Badger

Striped Skunk

River Otter

Lynx

Woodland Caribou

Mule Deer

White-tailed Deer

Deer species

Moose

Elk

Moose/Elk

Pronghorn

Totals seen/heard on count day

Total species seen/heard

Total species recorded by tracks

Total species otherwise recorded

Species recorded count period

Total species count period and day

\begin{tabular}{r|r|r|r|} 
& & & \\
& & & \\
& $\mathrm{t}$ & & \\
\hline & & 3 & \\
\hline
\end{tabular}

\begin{tabular}{l|l|l|l|l}
$\mathrm{t}$ & & & & \\
\hline
\end{tabular}

\begin{tabular}{|l|l|l|l|l|l|}
\hline 5 & & & & & \\
\hline & $\mathrm{t}$ & 1 & & 1 & $\mathrm{t}$
\end{tabular}

\begin{tabular}{ll|r|r|r|r|r}
\hline$t$ & $t$ & 2 & & & $t$ & \\
& & & 1 & 1 & &
\end{tabular}

\begin{tabular}{|r|r|r|r|r|r|r|r|r|r|r|r|}
\hline & & & & & & & & & & & \\
\hline 21 & 6 & & 6 & 10 & & & & 3 & 2 & 3 & 4 \\
\hline & & & & & & & & 4 & & & \\
\hline
\end{tabular}


Table 1. $30^{\text {th }}$ Saskatchewan Christmas Mammal Count-2002.

Species

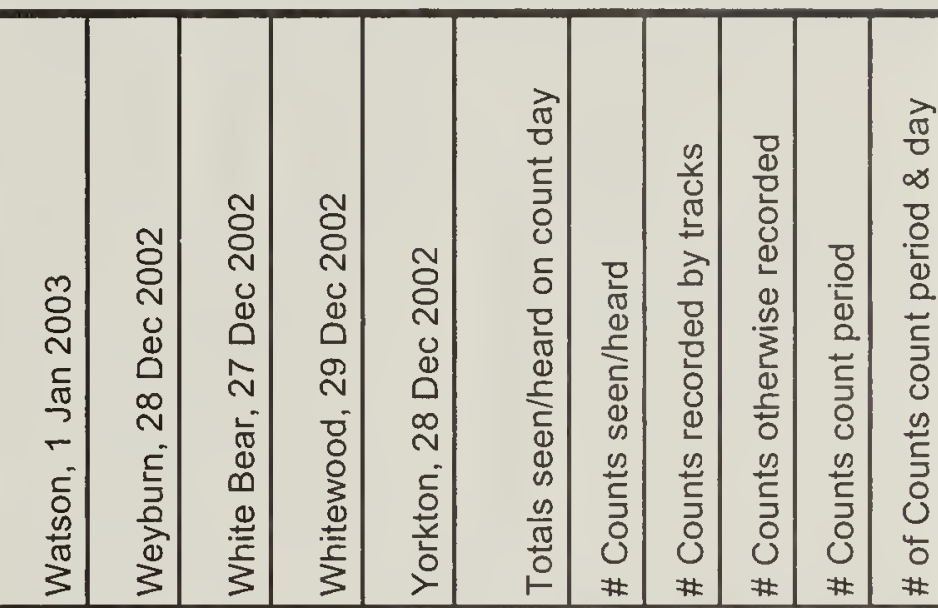

\section{Shrew species}

Nuttall's Cottontail

Snowshoe Hare

White-Tailed Jack Rabbit

Richardson's Ground Squirrel

Black-Tailed Prairie Dog

Grey Squirrel

Fox Squirrel

American Red Squirrel

Northern Flying-Squirrel

American Beaver

Deer Mouse

Muskrat

Meadow Vole

Vole species

Jumping Mouse species

Mouse species

Norway Rat

House Mouse

American Porcupine

Coyote

Wolf

Red Fox

Raccoon

American Marten

Fisher

\section{Ermine}

Long-tailed Wease!

Least Weasel

Weasel species

American Mink

American Badger

Striped Skunk

River Otter

Lynx

Woodland Caribou

Mule Deer

White-tailed Deer

Deer species

Moose

Elk

Moose/Elk

Pronghorn

Totals seen/heard on count day

Total species seen/heard

Total species recorded by tracks

Total species otherwise recorded

Species recorded count period

Total species count period and day

\begin{tabular}{r|r|r|r|r|r|r|r|}
\hline & & & & & & \\
\hline
\end{tabular}

\begin{tabular}{ll|l|l|l|l}
2 & 2 & 3 & 0 & 0 & 5
\end{tabular}

\begin{tabular}{r|r|r|r|r|r}
33 & 10 & 4 & 1 & 1 & 16 \\
\hline 43 & 13 & 32 & 0 & 0 & 45 \\
\hline
\end{tabular}

\begin{tabular}{r|r|r|r|r|r|r|r|r|r|r|}
\hline$t$ & 2 & 3 & 1 & & 81 & 17 & 15 & 0 & 0 & 32 \\
\hline & & & & & 1 & 1 & 0 & 0 & 1 & 2 \\
\hline
\end{tabular}

\begin{tabular}{|l|l|l|l|l|l|}
100 & 1 & 0 & 0 & 0 & 1 \\
17 & 6 & 0 & 0 & 0 & 6
\end{tabular}

\begin{tabular}{l|l|l|l|l|l|}
14 & 6 & 0 & 0 & 0 & 6 \\
\hline
\end{tabular}

\begin{tabular}{r|r|r|r|r|r}
406 & 51 & 1 & 0 & 0 & 52 \\
\hline
\end{tabular}

\begin{tabular}{r|r|r|r|r|r}
6 & 2 & 0 & 0 & 1 & 3 \\
\hline
\end{tabular}

\begin{tabular}{r|r|r|r|r|r|}
21 & 5 & 8 & 0 & 2 & 15 \\
\hline
\end{tabular}

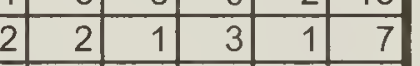

\begin{tabular}{|r|r|r|r|r|r|}
11 & 7 & 1 & 0 & 0 & 8 \\
\hline 14 & 1 & 12 & 0 & 0 & 13
\end{tabular}

\begin{tabular}{r|r|r|r|r|r|}
\hline 0 & 0 & 1 & 0 & 0 & 1 \\
\hline
\end{tabular}

\begin{tabular}{|r|r|r|r|r|r|}
\hline 3 & 1 & 10 & 0 & 0 & 11 \\
\hline
\end{tabular}

\begin{tabular}{l|r|r|r|r|r|}
\hline 0 & 0 & 1 & 0 & 0 & 1
\end{tabular}

\begin{tabular}{l|l|l|l|l|l|}
0 & 0 & 1 & 0 & 0 & 1 \\
\hline 6 & 2 & 1 & 0 & 0 & 3
\end{tabular}

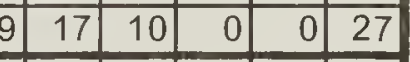

\begin{tabular}{|l|l|l|l|l|l|}
\hline 46 & 52 & 20 & 0 & 2 & 74 \\
\hline
\end{tabular}

\begin{tabular}{r|r|r|r|r|r|}
5 & 52 & 20 & 0 & 2 & 74 \\
\hline 9 & 3 & 5 & 0 & 0 & 8 \\
\hline
\end{tabular}

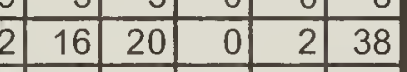

\begin{tabular}{r|r|r|r|r|r}
1 & 16 & 20 & 0 & 2 & 38 \\
\hline 1 & 1 & 6 & 0 & 0 & 7
\end{tabular}

\begin{tabular}{l|l|l|l|l|l|}
\hline 0 & 0 & 1 & 0 & 0 & 1 \\
\hline
\end{tabular}

\begin{tabular}{l|l|l|l|l|l|}
\hline 0 & 0 & 2 & 0 & 1 & 3 \\
\hline
\end{tabular}

\begin{tabular}{|r|r|r|r|r|r|}
\hline 1 & 1 & 10 & 0 & 2 & 13 \\
\hline 9 & 4 & 8 & 0 & 1 & 13 \\
\hline
\end{tabular}

\begin{tabular}{r|r|r|r|r|r|}
9 & 4 & 8 & 0 & 1 & 13 \\
\hline 1 & 1 & 4 & 0 & 1 & 6
\end{tabular}


Table 2. $31^{\text {st }}$ Saskatchewan Christmas Mammal Count-2003.

Species

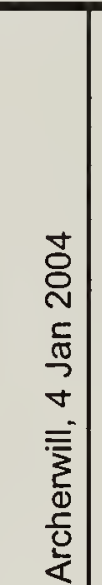

|

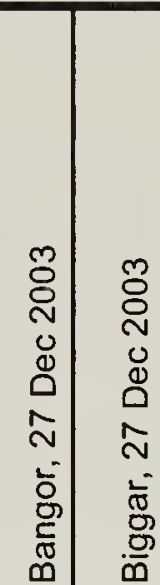

Shrew Species

Nuttall's Cottontail

Snowshoe Hare

White-tailed Jack Rabbit

Richardson's Ground Squirrel

13-lined Ground Squirrel

Least Chipmunk

Grey Squirrel

Fox Squirrel

American Red Squirrel

\begin{tabular}{l|l|l|l|l|l}
\hline & & & & \\
\hline & & & &
\end{tabular}

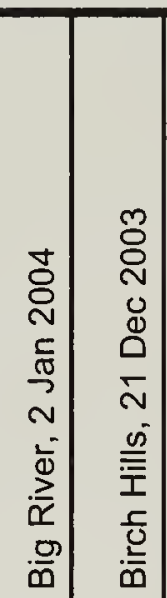

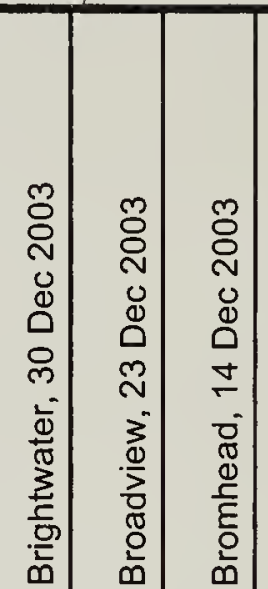

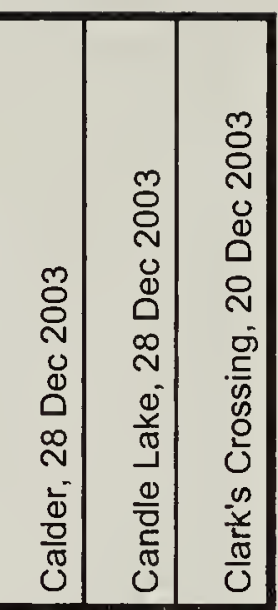

Northern Flying-Squirrel

American Beaver

Deer Mouse

Muskrat

Meadow Vole

Vole Species

Mouse Species

Norway Rat

House Mouse

American Porcupine

\begin{tabular}{|c|c|c|c|c|c|c|c|c|c|c|c|}
\hline & & & & & 2 & & $\mathrm{t}$ & & & t & $\mathrm{t}$ \\
\hline 1 & & 2 & & & & 1 & $t$ & $t$ & & & \\
\hline & & & & & & & & & & & \\
\hline
\end{tabular}

Coyote

Wolf

Red Fox

Swift Fox

Black Bear

Raccoon

American Marten

Fisher

Ermine

Long-tailed Weasel

Least Weasel

Weasel Species

American Mink

American Badger

Striped Skunk

Lynx

Mule Deer

White-tailed Deer

Deer Species

Moose

Elk

1

1

$+$

\begin{tabular}{r|r|r|r|r}
\hline & & & & \\
\hline & & & 1
\end{tabular}

$\mid$

$+$

\section{Pronghorn}

Totals seen/heard on count day

Total species seen/heard

\begin{tabular}{l|r|r|}
\hline 11 & 2 & 1
\end{tabular}

1

\begin{tabular}{l|r|r}
$\mathrm{t}$ & 1 \\
\hline
\end{tabular}

4

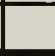

Total species recorded by tracks

Total species otherwise recorded

\begin{tabular}{ll|l|l|l|}
\hline & & & & \\
\hline & & & & \\
\hline & & & 0
\end{tabular}

\begin{tabular}{ll|l|l|}
\hline & & \\
\hline & & \\
\hline
\end{tabular}

\begin{tabular}{l|l}
\hline & \\
\hline & \\
\hline &
\end{tabular}

$\mid$

Species recorded count period

Total species count period and day 
Table 2. $31^{\text {st }}$ Saskatchewan Christmas Mammal Count-2003.

\section{Species}

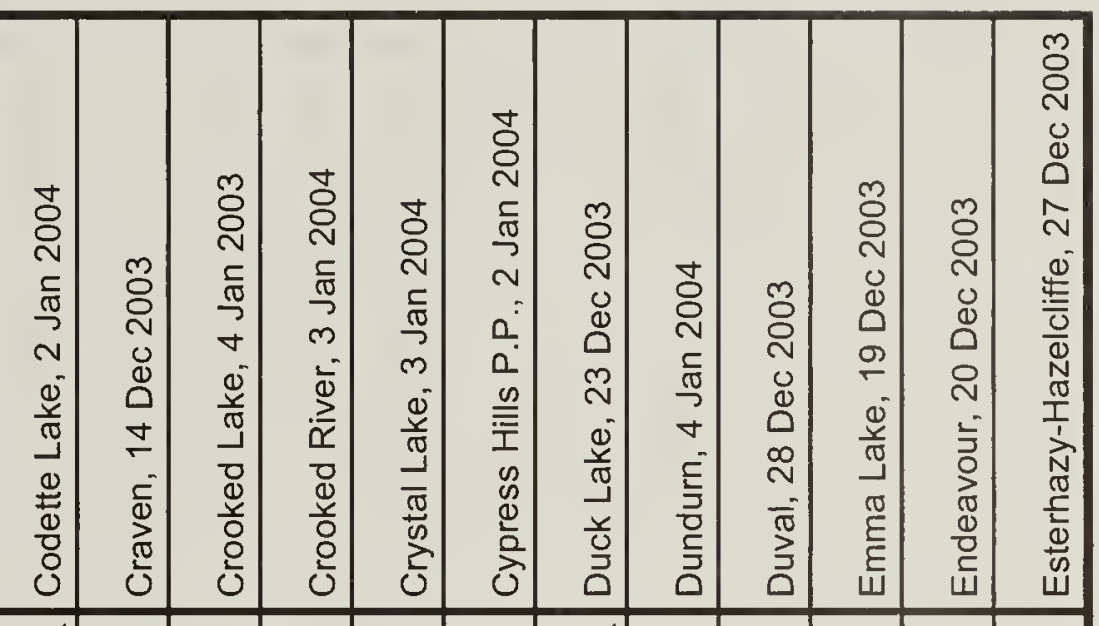

Shrew Species

Nuttall's Cottontail

Snowshoe Hare

White-tailed Jack Rabbit

Richardson's Ground Squirrel

13-lined Ground Squirrel

Least Chipmunk

Grey Squirrel

Fox Squirrel

American Red Squirrel

Northern Flying-Squirrel

American Beaver

Deer Mouse

Muskrat

Meadow Vole

Vole Species

Mouse Species

Norway Rat

House Mouse

American Porcupine

Coyote

Wolf

Red Fox

Swift Fox

Black Bear

Raccoon

American Marten

Fisher

Ermine

Long-tailed Weasel

Least Weasel

Weasel Species

American Mink

American Badger

Striped Skunk

River Otter

Lynx

Mule Deer

White-tailed Deer

Deer Species

Moose

Elk

Pronghorn

Totals seen/heard on count day

Total species seen/heard

Total species recorded by tracks

Total species otherwise recorded

Species recorded count period

Total species count period and day

\begin{tabular}{rr|r|r|r}
\hline$t$ & & & \\
\hline & $t$ & & & $t$ \\
\hline & $\mathrm{t}$ & &
\end{tabular}

1

t

\begin{tabular}{r|l|l|l|l|l|l|}
\hline 1 & & & & & \\
\hline & & & & & \\
\hline
\end{tabular}

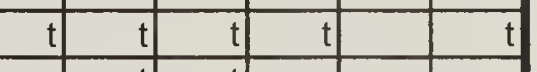

\begin{tabular}{|l|r|r|r|r|r|r|r|r|r|r|}
\hline 4 & 4 & 1 & & 4 & 10 & & & 14 & & 1 \\
\hline & & & & & & & & & & \\
\hline
\end{tabular}


Table 2. $31^{\text {st }}$ Saskatchewan Christmas Mammal Count-2003.

\section{Species}

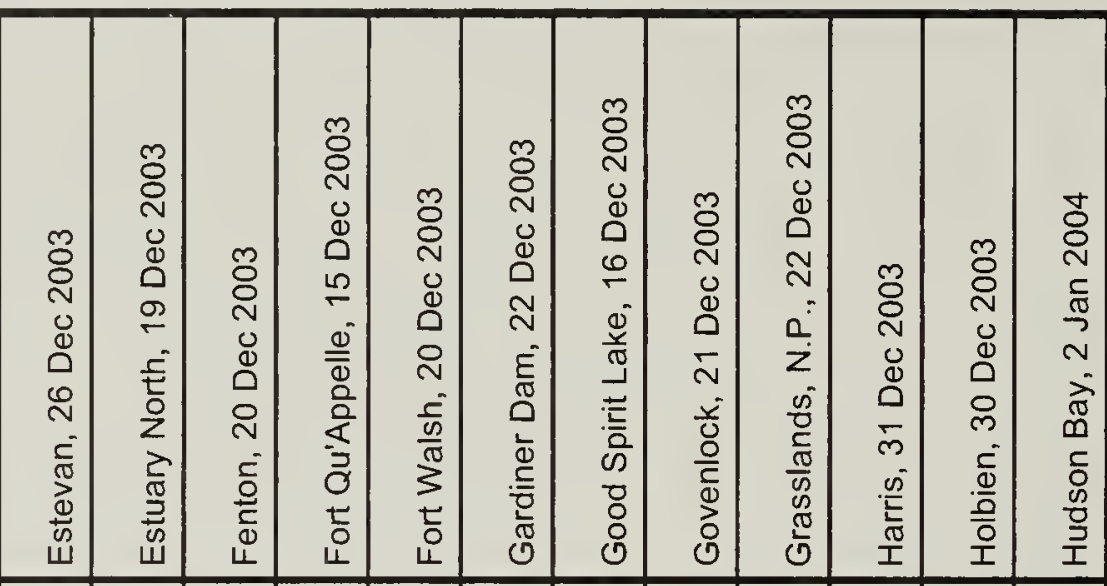

Shrew Species

Nuttall's Cottontail

Snowshoe Hare

White-tailed Jack Rabbit

Richardson's Ground Squirrel

13-lined Ground Squirrel

Least Chipmunk

Grey Squirrel

Fox Squirrel

American Red Squirrel

Northern Flying-Squirrel

American Beaver

Deer Mouse

Muskrat

Meadow Vole

Vole Species

Mouse Species

Norway Rat

House Mouse

American Porcupine

Coyote

Wolf

Red Fox

Swift Fox

Black Bear

Raccoon

American Marten

Fisher

Ermine

Long-tailed Weasel

Least Weasel

Weasel Species

\section{American Mink}

American Badger

Striped Skunk

River Otter

Lynx

Mule Deer

White-tailed Deer

Deer Species

Moose

Elk

Pronghorn

4

\begin{tabular}{r|r|r|r}
\hline 1 & 1 & 1 & \\
\hline 1 & $t$ & &
\end{tabular}

\begin{tabular}{l|l|l}
6 & 1 \\
\hline
\end{tabular}

\begin{tabular}{|r|r|r|r|r|r|r|}
\hline 6 & $t$ & 1 & $t$ & & $t$ & \\
\hline
\end{tabular}

Totals seen/heard on count day

Total species seen/heard

Total species recorded by tracks

Total species otherwise recorded

Species recorded count period

Total species count period and day

\begin{tabular}{l|l|r|}
\hline & & 6 \\
\hline & & \\
\hline
\end{tabular}

1

-

\begin{tabular}{rr|r|r|r|r|r|r}
\hline & & & & & & \\
\hline & 1 & & & & & \\
\hline & & & 4 & 7 & 13 & \\
\hline & & & & $\mathrm{t}$ & 1 & & \\
\hline & & & & 1 & & \\
\hline & & & & & & & \\
\hline
\end{tabular}

\begin{tabular}{|l|l|l|}
\hline & \\
\hline & &
\end{tabular}

\begin{tabular}{l|r|r|r} 
& $t$ & & \\
\hline & $t$ & & \\
\hline & & & \\
\hline & & & \\
\hline
\end{tabular}

\begin{tabular}{r|}
10 \\
\hline 2 \\
\hline
\end{tabular}

\begin{tabular}{r|r|r|r} 
& & & \\
\hline & & & \\
\hline
\end{tabular}

\begin{tabular}{|c|c|}
\hline \\
\hline 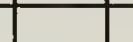 & \\
\hline & \\
\hline $\mathrm{t}$ & \\
\hline
\end{tabular}

\begin{tabular}{r|r|}
\hline & \\
\hline & \\
\hline & $\mathrm{t}$ \\
\hline
\end{tabular}

\begin{tabular}{ll} 
& \\
\hline$t$ & \\
\hline
\end{tabular}

\begin{tabular}{l|l}
\hline & \\
\hline & \\
\hline &
\end{tabular}

\begin{tabular}{rr|r|r|r|r|r|r|r|r|r|r}
\hline \\
\hline
\end{tabular}


Table 2. $31^{\text {st }}$ Saskatchewan Christmas Mammal Count-2003.

Species

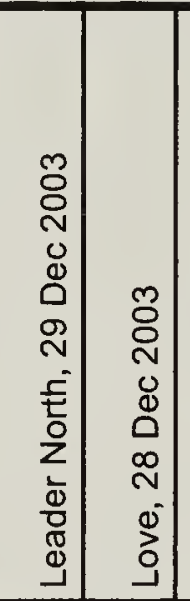

Shrew Species

Nuttall's Cottontail

Snowshoe Hare

White-tailed Jack Rabbit

Richardson's Ground Squirrel

13-lined Ground Squirrel

Least Chipmunk

Grey Squirrel

Fox Squirrel

American Red Squirrel

Northern Flying-Squirrel

American Beaver

Deer Mouse

Muskrat

Meadow Vole

Vole Species

Mouse Species

Norway Rat

House Mouse

American Porcupine

Coyote

Wolf

Red Fox

Swift Fox

Black Bear

Raccoon

American Marten

Fisher

Ermine

Long-tailed Weasel

Least Weasel

Weasel Species

American Mink

American Badger

Striped Skunk

River Otter

Lynx

Mule Deer

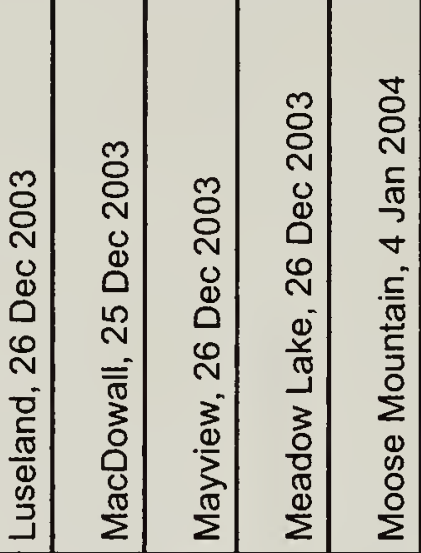

White-tailed Deer

Deer Species

Moose

Elk

Pronghorn

Totals seen/heard on count day

Total species seen/heard

Total species recorded by tracks

Total species otherwise recorded

\begin{tabular}{|r|r|r|}
\hline & & \\
\hline 1 & 2 & 1 \\
\hline & &
\end{tabular}

\begin{tabular}{r|r}
\hline$t$ & \\
\hline$t$ &
\end{tabular}

\begin{tabular}{|r|r|r}
\hline & & \\
\hline & &
\end{tabular}

1

\begin{tabular}{rr|r|r} 
& & & \\
\hline 1 & $c$ & $t$ & \\
\hline$t$ & $c$ & $t$ &
\end{tabular}

Species recorded count period

Total species count period and day

\begin{tabular}{lll|l|l|}
\hline & & & \\
\hline & & & \\
\hline
\end{tabular}

\begin{tabular}{ll|l}
\hline & & \\
\hline & &
\end{tabular}

\begin{tabular}{l|l}
\hline & \\
\hline & \\
\hline &
\end{tabular}

$\mathrm{t}$

\begin{tabular}{rr|r|r|r|r|r|r|r}
\hline & 14 & & 7 & 1 & 3 & 5 \\
\hline & & & & & 0 & \\
\hline & & & & & & \\
\hline & & & & & & \\
\hline & & & $\mathrm{t}$ & & $\mathrm{t}$ & \\
\hline & & & & & & \\
\hline & & & & & & \\
\hline
\end{tabular}

$+2$

\begin{tabular}{|rr|r|r|r|r|r|r|r|r|r|r|}
\hline 1 & $\mathrm{t}$ & & $\mathrm{t}$ & & & & $\mathrm{t}$ & & 1 & \\
\hline 4 & 2 & 4 & $\mathrm{t}$ & $\mathrm{t}$ & 1 & 3 & $\mathrm{t}$ & $\mathrm{c}$ & $\mathrm{o}$ & 20 \\
\hline & $\mathrm{t}$ & & & & $\mathrm{t}$ & & & & & & \\
\hline
\end{tabular}


Table 2. $31^{\text {st }}$ Saskatchewan Christmas Mammal Count-2003.

Species

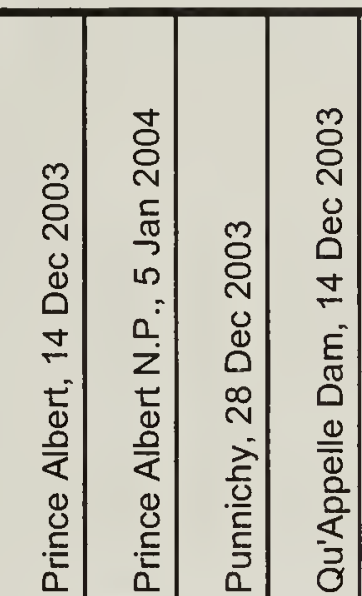

Shrew Species

Nuttall's Cottontail

Snowshoe Hare

White-tailed Jack Rabbit

Richardson's Ground Squirrel

13-lined Ground Squirrel

Least Chipmunk

Grey Squirre!

Fox Squirrel

American Red Squirre!

Northern Flying-Squirre!

American Beaver

Deer Mouse

Muskrat

Meadow Vole

Vole Species

Mouse Species

Norway Rat

House Mouse

American Porcupine

Coyote

Wolf

Red Fox

Swift Fox

Black Bear

Raccoon

American Marten

Fisher

Ermine

Long-tailed Weasel

Least Weasel

Weasel Species

American Mink

American Badger

Striped Skunk

River Otter

Lynx

Mule Deer

White-tailed Deer

Deer Species

Moose

Elk

Pronghorn

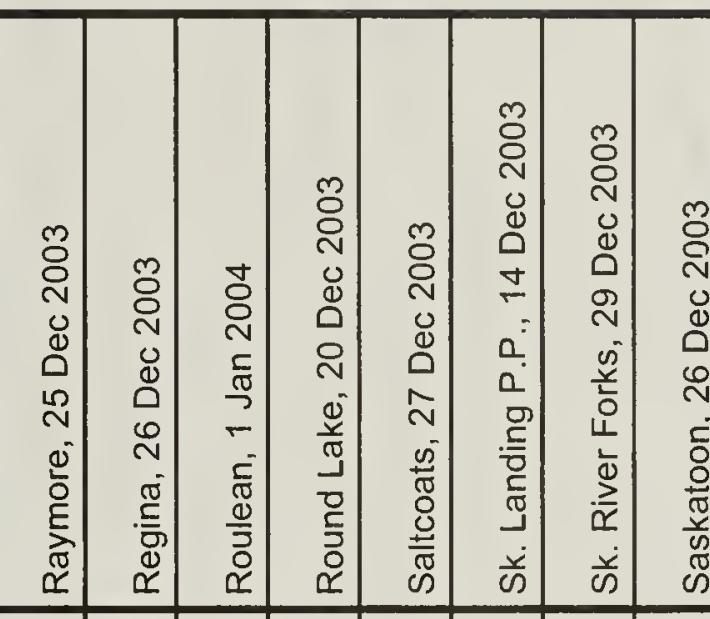

\begin{tabular}{rl|l|l|l|l|r|r|r|} 
& & & & & & $\mathrm{t}$ \\
\hline
\end{tabular}

\begin{tabular}{r|r|r}
-1 & \\
\hline
\end{tabular}

t

Totals seen/heard on count day

Total species seen/heard

1922

50

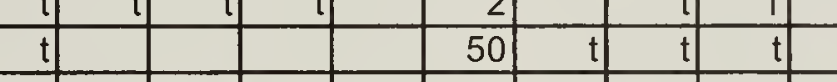

$+$

Total species recorded by tracks

Total species otherwise recorded

\begin{tabular}{|l|l|l|}
\hline & \\
\hline & & \\
\hline
\end{tabular}

\begin{tabular}{l|l|l|}
\hline & \\
\hline
\end{tabular}

\begin{tabular}{r|r|r|r|r} 
& & & & \\
\hline & & & & \\
\hline & & 6 & & \\
\hline
\end{tabular}

\begin{tabular}{rl|l|l|l|}
\hline & & & & \\
\hline & & & & $t$ \\
\hline & & & & \\
\hline
\end{tabular}

Species recorded count period

Total species count period and day

\begin{tabular}{rr|r|r|r|r|r} 
& 19 & 22 & & & & 2 \\
\hline & & & & & & \\
\hline & & & & & & 0 \\
\hline
\end{tabular}

\begin{tabular}{|r|r|r|r|r|r|r|}
\hline 2 & & 8 & 6 & & 8 & 5 \\
\hline $\mathrm{o}$ & & 0 & & 0 & & \\
\hline $\mathrm{t}$ & & & 1 & & & $\mathrm{t}$
\end{tabular}

\begin{tabular}{rr|r|r|r|l|l} 
& & & & 3 & & \\
\hline & & & & & & \\
& & & & & &
\end{tabular}

\begin{tabular}{l|l}
\hline $\mathrm{t}$ & \\
\hline & \\
\hline &
\end{tabular}

$+$

\begin{tabular}{ll|r|r|l|}
\hline & 1 & & \\
\hline & & & $\mathrm{t}$ & \\
\hline
\end{tabular}

\begin{tabular}{|r|r|r|r|r|r|r|r|r|r|r|r|}
\hline 1 & $\mathrm{t}$ & & 1 & & 2 & & & 1 & 2 & $\mathrm{t}$ & 2 \\
\hline 6 & $\mathrm{t}$ & 1 & 3 & & 4 & $\mathrm{c}$ & $\mathrm{t}$ & $\mathrm{t}$ & 3 & $\mathrm{t}$ & 2 \\
\hline & $\mathrm{t}$ & & & & & & & & & & \\
\hline
\end{tabular}


Table 2. $31^{\text {st }}$ Saskatchewan Christmas Mammal Count-2003.

Species

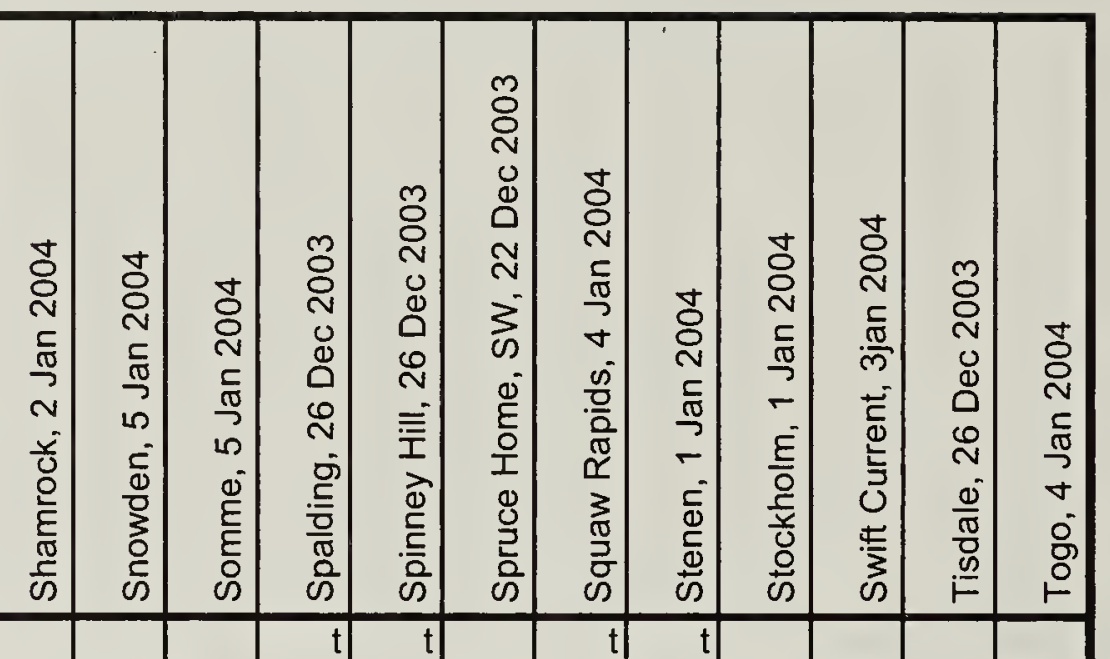

Shrew Species

Nuttall's Cottontail

Snowshoe Hare

White-tailed Jack Rabbit

Richardson's Ground Squirrel

13-lined Ground Squirrel

Least Chipmunk

Grey Squirrel

Fox Squirrel

American Red Squirrel

Northern Flying-Squirrel

American Beaver

Deer Mouse

Muskrat

Meadow Vole

Vole Species

Mouse Species

Norway Rat

House Mouse

American Porcupine

Coyote

Wolf

Red Fox

Swift Fox

Black Bear

Raccoon

American Marten

Fisher

Ermine

Long-tailed Weasel

Least Weasel

Weasel Species

American Mink

American Badger

Striped Skunk

River Otter

Lynx

Mule Deer

White-tailed Deer

Deer Species

Moose

Elk

Pronghorn

Totals seen/heard on count day

Total species seen/heard

Total species recorded by tracks

Total species otherwise recorded

Species recorded count period

Total species count period and day

\begin{tabular}{rrr|r|r|r|r|r|} 
& & & & $\mathrm{t}$ & $\mathrm{t}$ & & \\
\hline & & $\mathrm{t}$ & & $\mathrm{t}$ & & $\mathrm{t}$ & $\mathrm{t}$ \\
\hline & & 3 & $\mathrm{c}$ & & $\mathrm{t}$ & & \\
\end{tabular}

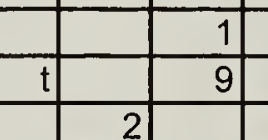

$+$


Table 2. $31^{\text {st }}$ Saskatchewan Christmas Mammal Count-2003.

Species

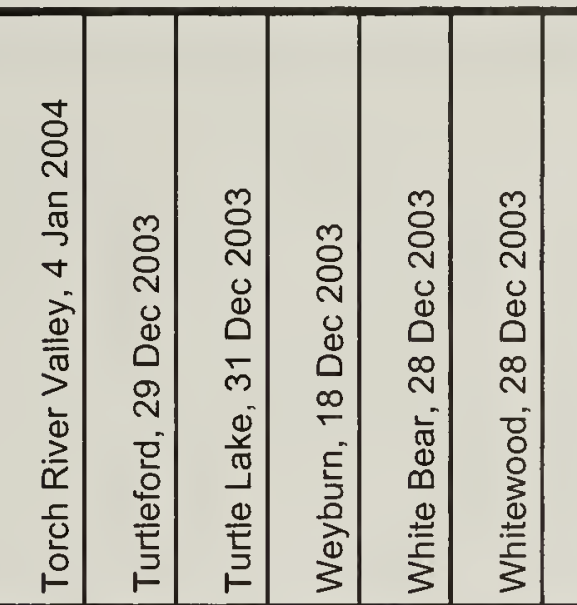

Shrew Species

Nuttall's Cottontail

Snowshoe Hare

White-tailed Jack Rabbit

Richardson's Ground Squirrel

13-lined Ground Squirrel

Least Chipmunk

Grey Squirrel

Fox Squirrel

American Red Squirrel

Northern Flying-Squirrel

American Beaver

Deer Mouse

Muskrat

Meadow Vole

Vole Species

Mouse Species

Norway Rat

House Mouse

American Porcupine

Coyote

Wolf

Red Fox

Swift Fox

Black Bear

Raccoon

American Marten

Fisher

Ermine

Long-tailed Weasel

Least Weasel

Weasel Species

American Mink

American Badger

Striped Skunk

River Otter

Lynx

Mule Deer

White-tailed Deer

Deer Species

Moose

Elk

Pronghorn

Totals seen/heard on count day

Total species seen/heard

Total species recorded by tracks

Total species otherwise recorded

Species recorded count period

Total species count period and day

3

\begin{tabular}{l|l|l|l|}
\hline & & \\
\hline
\end{tabular}

m.|.

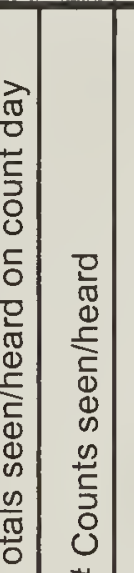

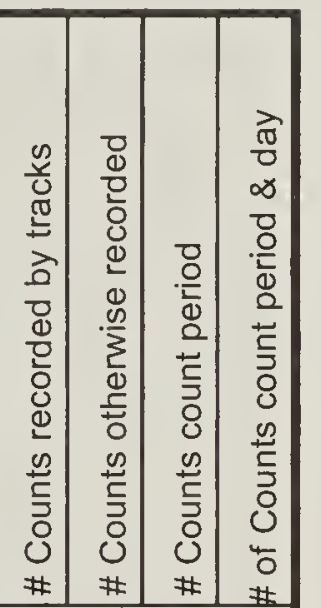

\begin{tabular}{rl|r|r|r|r|r|r|r|}
\hline 1 & & & 5 & 2 & 12 & 0 & 0 & 14 \\
\hline
\end{tabular}

\begin{tabular}{rl|r|r|r|r|r|r|r|r|r|}
$\mathrm{t}$ & & 1 & & & 24 & 9 & 0 & 0 & 0 & $\mathrm{~g}$ \\
\hline
\end{tabular}


Table 3. Summary of Saskatchewan Christmas Mammal Counts-1973-2003.

\begin{tabular}{|c|c|c|c|c|c|c|c|}
\hline \multirow[t]{2}{*}{ Year } & \multirow[t]{2}{*}{ Counts } & \multicolumn{5}{|c|}{ Species by category* } & \multirow[t]{2}{*}{ New Species } \\
\hline & & $\mathbf{s}$ & $\mathbf{t}$ & $\mathbf{0}$ & c & Total & \\
\hline 1974 & 16 & 12 & 2 & 0 & 1 & 15 & $\begin{array}{l}\text { Snowshoe Hare, White-tailed Jack } \\
\text { Rabbit, Grey Squirrel, Am. Red } \\
\text { Squirrel, Am. Porcupine, Coyote, Red } \\
\text { Fox, White-tailed Deer. } \\
\text { Am. Beaver, Deer Mouse, Meadow } \\
\text { Vole, Mule Deer. }\end{array}$ \\
\hline 1975 & 28 & 14 & 6 & 0 & 1 & 21 & $\begin{array}{l}\text { Franklin's Gr. Squirrel, Muskrat, } \\
\text { House Mouse, Long-tailed Weasel. }\end{array}$ \\
\hline 1976 & 33 & 14 & 8 & 0 & 1 & 23 & $\begin{array}{l}\text { Nuttall's Cottontail, Moose, Elk, } \\
\text { Pronghorn. }\end{array}$ \\
\hline 1977 & 41 & 13 & 6 & 2 & 3 & 24 & Least Weasel, Am. Badger. \\
\hline 1978 & 42 & 20 & 6 & 0 & 3 & 29 & Raccoon, American Mink, Bobcat. \\
\hline 1979 & 42 & 23 & 3 & 0 & 1 & 27 & Wolf, Striped Skunk. \\
\hline 1980 & 45 & 22 & 4 & 0 & 2 & 28 & $\begin{array}{l}\text { Richardson's Gr. Squirrel, Fox } \\
\text { Squirrel, Ermine. }\end{array}$ \\
\hline 1981 & 37 & 20 & 4 & 1 & 2 & 27 & \\
\hline 1982 & 49 & 22 & 3 & 2 & 1 & 28 & Northern Flying-Squirrel. \\
\hline 1983 & 55 & 25 & 5 & 1 & 1 & 32 & \\
\hline 1984 & 54 & 25 & 7 & 2 & 0 & 34 & Gapper's Red-Backed Vole, Fisher. \\
\hline 1985 & 62 & 27 & 3 & 0 & 2 & 32 & River Otter. \\
\hline 1986 & 66 & 30 & 4 & 0 & 3 & 37 & $\begin{array}{l}\text { Pygmy Shrew, Norway Rat, Am. } \\
\text { Marten. }\end{array}$ \\
\hline 1987 & 70 & 28 & 3 & 1 & 5 & 37 & Masked Shrew, Eastern Cottontail. \\
\hline 1988 & 68 & 28 & 1 & 2 & 5 & 36 & Woodchuck, Woodland Caribou. \\
\hline 1989 & 68 & 29 & 0 & 1 & 3 & 33 & (Little Brown Bat-dead, count period). \\
\hline 1990 & 65 & 28 & 5 & 2 & 3 & 38 & $\begin{array}{l}\text { Northern Pocket Gopher, (Big Brown } \\
\text { Bat-dead, count period). }\end{array}$ \\
\hline 1991 & 82 & 33 & 4 & 2 & 3 & 42 & $\begin{array}{l}\text { Short-tailed Shrew, Black-Tailed } \\
\text { Prairie Dog, (Sagebrush Vole-dead). }\end{array}$ \\
\hline 1992 & 69 & 22 & 8 & 1 & 5 & 36 & (Am. Water Shrew-count period). \\
\hline 1993 & 72 & 27 & 5 & 2 & 2 & 36 & \\
\hline 1994 & 84 & 32 & 7 & 0 & 1 & 40 & (Mountain Lion-tracks). \\
\hline 1995 & 78 & 33 & 4 & 0 & 1 & 38 & Swift Fox. \\
\hline 1996 & 82 & 29 & 3 & 1 & 4 & 37 & Black Bear, Lynx. \\
\hline 1997 & 96 & 32 & 4 & 0 & 3 & 39 & \\
\hline 1998 & 87 & 27 & 7 & 3 & 1 & 38 & \\
\hline 1999 & 94 & 30 & 3 & 0 & 3 & 36 & \\
\hline 2000 & 93 & 30 & 2 & 1 & 5 & 38 & \\
\hline 2001 & 102 & 32 & 5 & 0 & 5 & 42 & \\
\hline 2002 & 93 & 33 & 4 & 0 & 1 & 38 & (jumping mouse species-tracks). \\
\hline 2003 & 91 & 35 & 4 & 0 & 0 & 39 & 13-lined Gr. Squirrel, L. Chipmunk. \\
\hline Totals & 1972 & 49 & 2 & 0 & 4 & 55 & \\
\hline
\end{tabular}

$\mathrm{s}=$ seen, $\mathrm{t}=$ identified by tracks, $\mathrm{o}=$ identified by other methods (see introduction), $\mathrm{c}=$ recorded during count period but not on count day. 


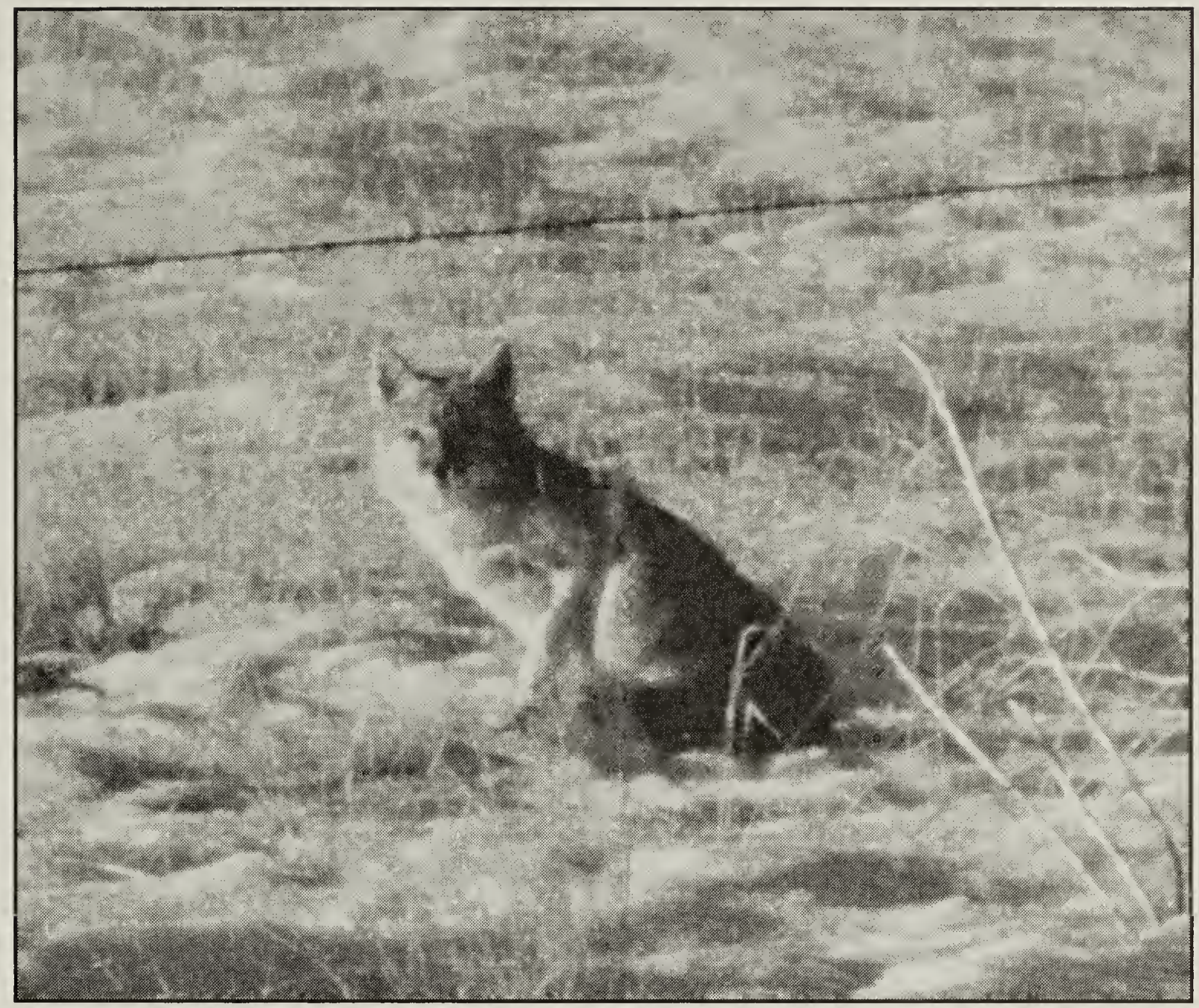

Swift Fox photographed on 21 December 2003 during the Christmas Mammal Count at Govenlock.

Val Harris

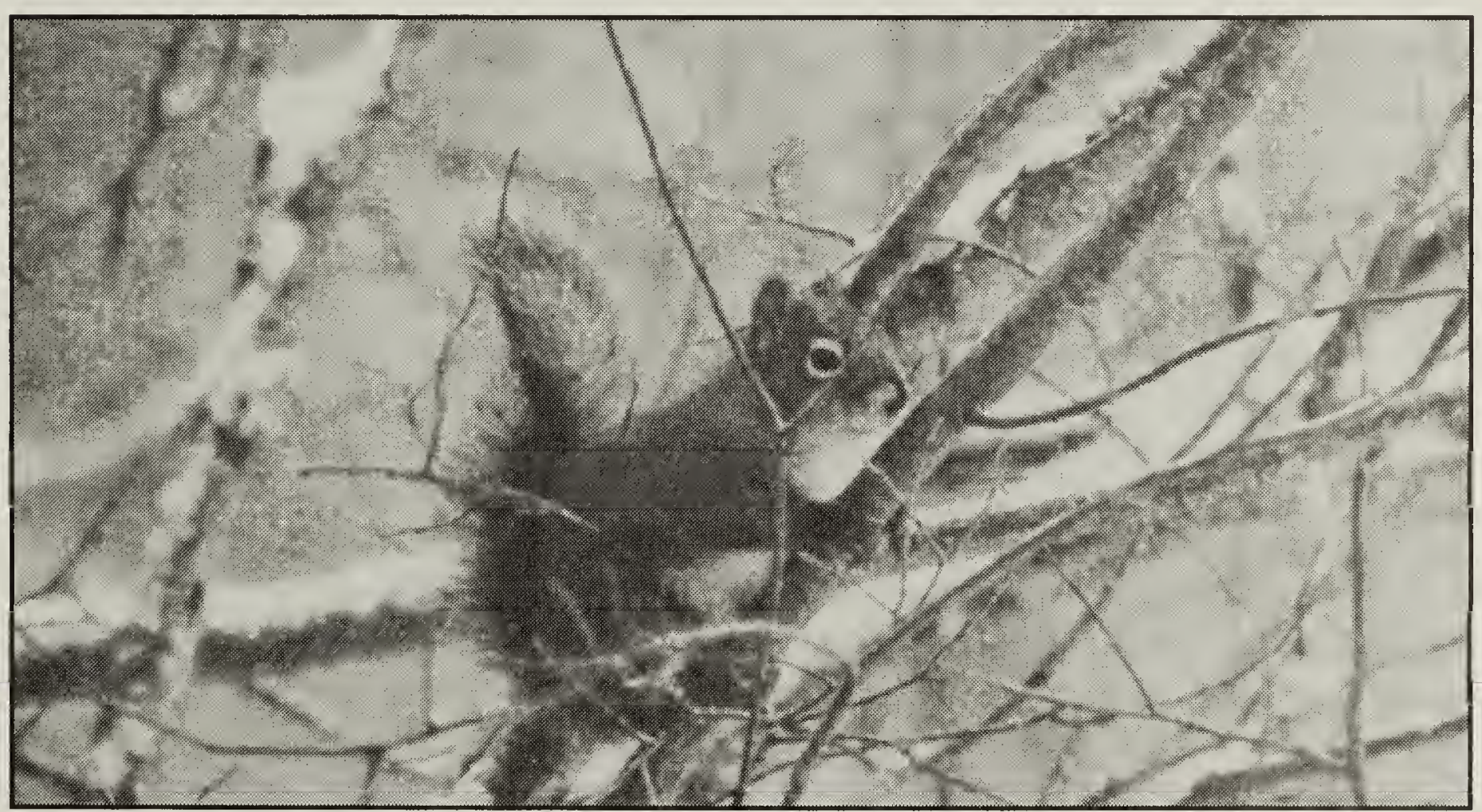

American Red Squirrel in winter.

Larry A. Morgotch 